\title{
GUTE PLATTFORM
}

\section{Liebe Leserin, lieber Leser,}

diese Sonderausgabe der ATZelektronik startet mit einem herzlichen Glückwunsch an die Messe München für 50 Jahre Electronica - eine Erfolgsstory, an der die Automobilbranche und vor allem unserer Magazin im neunten Jahrgang nur einen bescheidenen Anteil haben.

Umso mehr freut es uns sehr, dass die ATZelektronik als Premiumpartner für das Diskussionsforum während der diesjährigen Messe exklusiv für die Automobilbranche ausgewählt wurde. Unser gemeinsamer Anspruch ist, erstmals nicht Fachvorträge zu moderieren und nur Fragen zu ergänzen, sondern ausschließlich Brennpunktthemen in Podiumsdiskussionen und Interviews kritisch anzusprechen.

Zu einem der Brennpunktthemen zählt die nicht automobilspezifische Integration von Consumer-Elektronik in Fahrzeuge. Die Diskussion der bereits entstandenen Risiken und technischen wie auch rechtlichen Auswirkungen führt die Redaktion der ATZelektronik bereits seit dem Frühjahr dieses Jahres in mehreren Veröffentlichungen, unter anderem gemeinsam mit dem Zentralverband der Elektroindustrie, ZVEI. Im Artikel auf Seite 14 wird auf einige dieser Veröffentlichungen hingewiesen. Im Forum auf der Electronica wird nun die redaktionelle Dramaturgie deutlich, die wir in Richtung des Messe-Events aufgebaut haben. Die Auseinandersetzung mit dem CE-Thema am 12. November ist zunächst ein Highlight, weil sie mit vielen weiteren Hintergrundinformationen für die gewünschte Aufklärung sorgt.

Diskussionspartner ist unter anderem Helmut Keller, der in der ATZelektronikAprilausgabe mit dem Artikel „Consumer-Elektronik - bei funktionaler Sicherheit wird es kritisch“ auf Missstände aufmerksam machte. Der Sprecher des Arbeitskreises Robustness Validation beim ZVEI und pensionierte Werkleiter der Bosch-Halbleiterfertigung sensibilisiert in diesem Artikel für weitere heikle Aspekte, rechtliche Konsequenzen und die Produkthaftung der verantwortlichen Entwicklungsingenieure. Den Anwalt Andreas Reuter haben wir deswegen zu Diskussion zum Forum eingeladen, ergänzend zu seinen Veröffentlichungen in ATZelektronik.

Welche Konsequenzen sind in der Entwicklung von Fahrerassistenzsystemen speziell in den relevanten Safety und Security-Themenfeldern zu erwarten? Hierzu befragen wir im Automobilforum auf der Electronica Dr. Thomas Wollinger von Escrypt. Er ist Security-Experte und verfügt über Know-how aus anderen Branchen.

Branchenübergreifend. Das ist ein bezeichnendes Stichwort, das auf die willkommene Zusammenarbeit mit der Electronica passt. Die Messe hat den Fahrzeugsektor in München Jahr für Jahr stärker ausgebaut. Und nun ist die Autoindustrie gefragt, sich anderen Branchen zu öffnen. Somit gewinnt die Electronica in der Automobilbranche noch höhere Aufmerksamkeit.

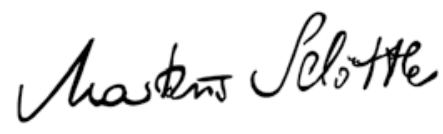

MARKUS SCHÖTTLE,

Stellvertretender Chefredakteur Wiesbaden, 6. Oktober 2014

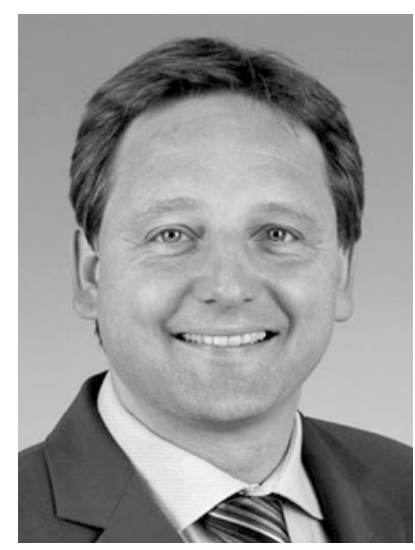

Internationale Messe und Konferenz für Leistungselektronik, Intelligente Antriebstechnik, Erneuerbare Energie und Energiemanagement

Nürnberg, 19. - 21.05.2015

\section{Mehr Power für Elektronik- PCIM Europe!}

\section{Ihr Marktplatz für Leistungselektronik}

